\title{
Modelling Ant Brood Tending Behavior with Cellular Automata
}

\author{
Daniel Merkle, Martin Middendorf, and Alexander Scheidler \\ Department of Computer Science, University of Leipzig, \\ Augustusplatz 10-11, D-04109 Leipzig, Germany \\ \{merkle, middendorf, scheidler\}@informatik.uni-leipzig.de
}

\begin{abstract}
The brood sorting behavior of ants like Leptothorax unifasciatus leads to patterns, where brood items are sorted in concentric circles around the nest center. The underlying mechanisms are not fully understood so far and brood tending simulations can help to explain the occurrence of these patterns. We reexamine an existing cellular automata based model for ant brood tending. This model is then modified and extended by including a carbon dioxide distribution in the nest, that influences the ants movement behavior. Furthermore, the ants can deliver food to the brood in our model. Results of simulation runs are presented that can help to explain brood patterns that have been observed in natural ant colonies.
\end{abstract}

\section{Introduction}

Brood sorting and brood tending behavior in ants has inspired several novel methods in computer science like clustering algorithms or multi robot systems (e.g., [2]). The underlying mechanisms which lead to such complex behavior are still under investigation. In 3 the pattern formation within Leptothorax unifasciatus ant colonies was investigated in artificial nests. The youngest brood items (eggs and microlarvae) are placed in the center, successively larger larvae are arranged in concentric rings around the center. However, the largest and oldest brood (pupae and prepupae) is placed in an intermediate area between the peripheral larvae and the larvae of medium size. One suggestion why this happens is that these patterns help to organize the brood care [3. The most valuable brood has to be fed first and is therefore placed at the outside, the pupae and prepupae may be placed in intermediate positions as they do not need food but only grooming.

In the inspiring paper [7] a stochastic lattice gas model of ant brood tending was formulated. The authors suggested, that a possible reason for the central egg location is that brood care is more evenly distributed in that area. We reexamine this model, make modifications to avoid unwanted artifacts, and extend it by taking a $\mathrm{CO}_{2}$ distribution in the nest into account that influences the ants movement behavior. Moroever, we simulate the brood feeding behaviour of the ants. The paper is structured as follows. Section 2 reviews the chemical 
and biological background which is needed in the models. The cellular automata transition functions used in [7] and our modifications are presented in Section 3] The model and the results from [7] are reexamined in Section 4, Experimental results with our model are discussed in Section 5 and a conclusion is given in Section 6 .

\section{Biological Background}

Several studies have shown the influence of carbon dioxide $\left(\mathrm{CO}_{2}\right)$ concentration on the behavior of social insects (e.g., 6 ). In 1 a diffusion model was introduced and spatial patterns of $\mathrm{CO}_{2}$ concentration were explored analytically and numerically. A special role plays the concentration gradient, as it is qualitatively independent of fluctuations of the absolute concentration. Ants are likely to have the ability to detect this gradient with their antenna and are able to infer regions of $\mathrm{CO}_{2}$ sources (e.g., nest center) and $\mathrm{CO}_{2}$ sinks (e.g., the nest entrance or periphery). In 8 the ability to detect the direction of the colony center was used to model worker sorting in ant colonies. This approach was based on a model in [4] for bacteria moving along a chemical gradient.

Besides the knowledge of the nest center the ants in our model will also carry food. Ants carrying food tend to move towards the nest center, ants without food tend to move to the periphery of the nest. Each ant will drop the food with a certain probability.

\section{Transition Functions and Measures for Brood Care}

This section describes the transition functions of the cellular automata models which are investigated in this paper. For all models the two dimensional Moore neighborhood $N=\{(-1,1), \ldots,(1,1)\}$ and a space $R=\{1, \ldots, L\} \times\{1, \ldots, L\}$ is used. At any lattice site there can only be 0 or 1 ant. In all models we proceed similar to the cellular automata model in [7]: Within one time step all ants move asynchronously and in random order. If an ant wants to move onto a lattice site, that is occupied by another ant, it will not move. The driving rate $p$ is the probability that ants occur on the boundary square, an ant which moves onto a boundary lattice is deemed to have left the brood, and is removed. Note, that the following description of the ants movement behavior could easily be transformed into a stochastic transition function for a cellular automata.

\subsection{Transition Functions for Modelling Ant Movement Behavior}

Deterministic movement: The deterministic behavior of the ants as described in 7] is based on the transition function used in [5]. An ant tries to move in a direction which takes it as far as possible from the other ants of the four neighboured cells (north, south, west and east). Formally, let $n$ be the preferred direction of motion for an ant, which is determined as $n=\left[F_{x} / F\right] i+\left[F_{y} / F\right] j$ where $F_{x}=a(r-i, t)-a(r+i, t), F_{y}=a(r-j, t)-a(r+j, t)$, and $F=\sqrt{F_{x}^{2}+F_{y}^{2}}$, 
$i$ and $j$ are the standard basis vectors in two dimensional space, operator $[x]$ means the nearest integer to $x, a(r, t)$ is the number of ants located in cell $r$ at time $t$. In [7] the tendency of ants to move in direction to the nest center is modelled by placing a ring of stationary ants around the actual space $R$, thus an ant which occurs on a boundary square of $R$ will be forced to move away from the boundary (a similar strategy was used in [5]).

Probabilistic movement behavior: To incorporate stochastic elements to the ants behavior the following strategy was suggested in [7. Let $n$ be the preferred direction according to the deterministic behavior of the ant, and $d\left(n^{*}\right)=\left|n^{*}-n\right|$ be a function that quantifies the deviation from direction $n \in N$ to a direction $n^{*} \in N$. The probability $P^{n, \beta}$ of moving to $n^{*}$ is calculated as

$$
P^{n, \beta}\left(n^{*}\right)=\frac{e^{-\beta d\left(n^{*}\right)}}{\sum_{n^{*} \in N} e^{-\beta d\left(n^{*}\right)}}
$$

where $\beta$ is a parameter that determines the degree of randomness of the movement direction of an ant. If $\beta=0$ all directions are equally likely, if $\beta \rightarrow \infty$ the probability distribution in Equation 1 approaches a delta function peaked at $n^{*}=n$, i.e., ants behave according to the deterministic movement.

Brood Care Measure: It is argued in [7] that brood tending is of less quality, if brood care is unevenly distributed. Therefore, let $\tau(r, t)$ be the amount of brood care (i.e., how often an ant was located at cell $r$ within the first $t$ steps), let $\bar{\tau}(t)=1 / L^{2} \sum_{r \in R} \tau(r, t)$ be the mean tending time per brood item, and $\sigma(t)=\left[1 / L^{2} \sum_{r \in R}(\tau(r, t)-\bar{\tau}(t))^{2}\right]^{1 / 2}$ be the standard deviation of brood tending times. Then the relative fluctuation amplitude of brood tending times, $\sigma^{*}(t)=\sigma(t) / \bar{\tau}(t)$ is a dimensionless measure of fluctuations in the amount of brood care. A value of $\sigma^{*}(t)<0.1$ is considered as efficient brood tending.

\subsection{Transition Function for the Extended Model}

In this subsection we will present our extended model for ant brood tending which incorporates a $\mathrm{CO}_{2}$ distribution within the nest and the ability of ants to deliver food. Two new parameters are $\sigma$ for the standard deviation of the two dimensional normal distribution, that is used for modelling the $\mathrm{CO}_{2}$ pattern within the nest, and parameter $f$ which is the probability that a food carrying ant drops it.

Strict gradient movement: For modelling the movement of ants towards or away from the nest center, we determine $\mathrm{CO}_{2}$ levels in the nest according to a two dimensional normal distribution. The $\mathrm{CO}_{2}$ level in cell $r=(x, y)$ is

$$
g_{r}=\frac{1}{2 \pi \sigma^{2}} \cdot e^{-\frac{1}{2}\left(\left(\frac{x-\mathbf{c}}{\sigma L}\right)^{2}+\left(\frac{y-\mathbf{c}}{\sigma L}\right)^{2}\right)}
$$

where $c=(\mathbf{c}, \mathbf{c})$ with $\mathbf{c}=\frac{L+1}{2}$ is the center of the nest. An ant uses the gradient of these $\mathrm{CO}_{2}$ levels to determine its movement. An ant that carries food is moving 
towards the center of the nest, an ant without food is moving away from the center. Therefore, we use $I=\{(1,0),(0,1),(1,1),(-1,1)\}$ for determining the pheromone gradient sum $G_{r}=\sum_{i \in I}\left|g_{r+i}-g_{r-i}\right|$, and let $G^{\max }=\max _{r \in R} G_{r}$ be the maximal gradient sum in the nest, which will be used for normalization of the probabilistic behavior. Formally, the probability that an ant carrying food located at cell $r$ will move in direction $n \in N$ is determined as follows:

$$
P_{r}^{g}(n)= \begin{cases}\frac{\left|g_{r+n}-g_{r-n}\right|}{G^{\max }} & \text { if } n \neq(0,0) \text { and } g_{r+n}>g_{r-n} \\ 1-\sum_{m \in N \backslash(0,0)} P_{r}^{g}(m) & \text { if } n=(0,0) \\ 0 & \text { otherwise }\end{cases}
$$

For an ant without food the same formula is used, but in the first case $g_{r+n}>$ $g_{r-n}$ has to be exchanged by $g_{r+n}<g_{r-n}$. Note, that the probability to move is small (resp. large) in areas where the sum of gradients of the $\mathrm{CO}_{2}$ level is small (resp. large).

Probabilistic movement behavior: To incorporate more randomness in the ants behavior which move according to the strict gradient movement, we used two methods. The first method is according to [7]. In contrast to the incorporation of randomness as given in Equation 1, there is no preferred direction $n$ of an ant (which is used to calculate the probabilities $P^{n, \beta}$ ). Instead a probability vector $P_{r}^{g}$ is used, that determines the probabilities that an ant located at cell $r$ moves to a certain neighbor cell when strict gradient movement behavior is used. $P^{n_{1}, \beta}\left(n_{2}\right)$ is the probability that an ant with the preferred movement direction $n_{1}$ moves in direction $n_{2}$ (see Equation 11). Then

$$
P_{r}(n)=\sum_{\substack{\left(n_{1}, n_{2}\right) \\ n_{1}+n_{2}=n}} P_{r}^{g}\left(n_{1}\right) \cdot P^{n_{1}, \beta}\left(n_{2}\right)
$$

determines the probability that an ant located at cell $s$ moves to neighbor $n$. Similar to Equation 1 each neighbor is equally likely for $\beta=0$, and for $\beta \rightarrow \infty$ we have $P_{r}(n)=P_{r}^{g}(n)$, i.e., the ants behave according to the strict gradient movement.

The second method to incorporate more randomness simply uses a linear combination of the strict gradient movement behavior and a pure random behavior, i.e.

$$
P_{r}(n)=(1-\lambda) P_{r}^{g}(n)+\lambda(1 /|N|)
$$

where $\lambda$ determines the degree of randomness $(\lambda=0$ leads to a strict gradient movement behavior, and for $\lambda=1$ a movement to any neighbor is equally likely).

Brood Care Measure: As suggested in [7] we count the number of brood tending times $\tau(r, t)$ of brood located at cell $r$ within $t$ steps. As in this model ants carry food, we will also measure the number $\zeta(r, t)$ of feeding times, i.e. how often food is dropped in cell $r$ within $t$ steps. For our investigations we will also use the mean tending time per brood member in certain areas around the center $c=(\mathbf{c}, \mathbf{c})$ of the nest. Therefore, we measure $\overline{\tau_{R}}(t, k)=1 /\left|R_{k}\right| \sum_{r \in R_{k}} \tau(r, t)$ 
with $R_{k}=\{r \in R: k-1 \leq\|r-c\| \leq k\}, k \geq 1$. If for $\|r-c\|$ the $L 2$ norm is used, this function is denoted by $\bar{\tau}_{\text {circ }}(t, k)$, if the $L$-infinity norm is used, it is denoted by $\bar{\tau}_{\text {square }}(t, k)$. We proceed similar for mean feeding times $\bar{\zeta}(t, k)$.

\section{Reexamination of a Brood Tending Model}

In this section we reexamine the results presented [7]. Brood care $\sigma^{*}$ was measured on a field of size $L=40$ for different degrees of randomness $(\beta \in\{0$, $0.1,0.5,1,3,5, \infty\})$ and different driving rates $p \in\{0.05,0.1,0.2,0.5,1\}$. The brood care intensity values for the whole space and for the center of the brood (the central $12 \times 12$ lattice) are given in Figure 1 .

In our simulation we basically obtained the same results as presented in [7, but we can not agree with their interpretation why the brood care is worse at the periphery. We consider this mainly as an artifact of the model. To show this
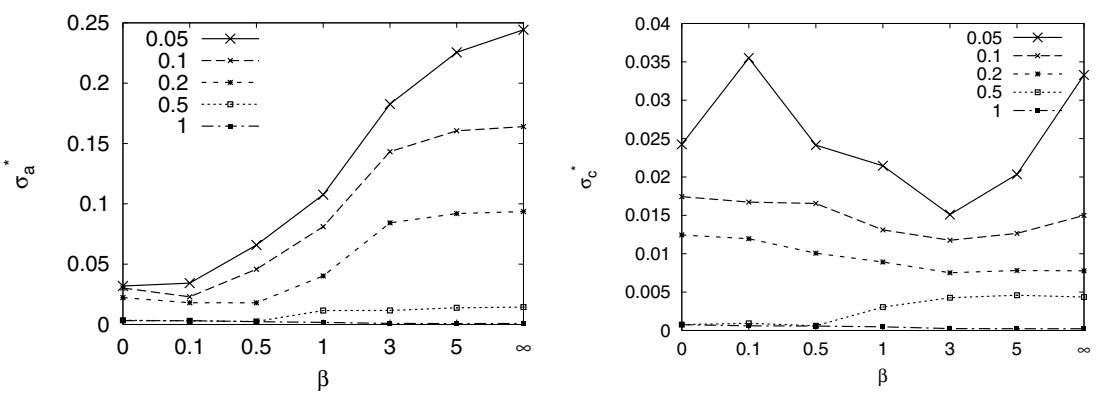

Fig. 1. Asymptotic relative fluctuation $\sigma^{*}$ for different values of $\beta$ and $p$ (different curves) in the whole nest of size $40 \times 40$ (left) and in the center square of size $12 \times 12$
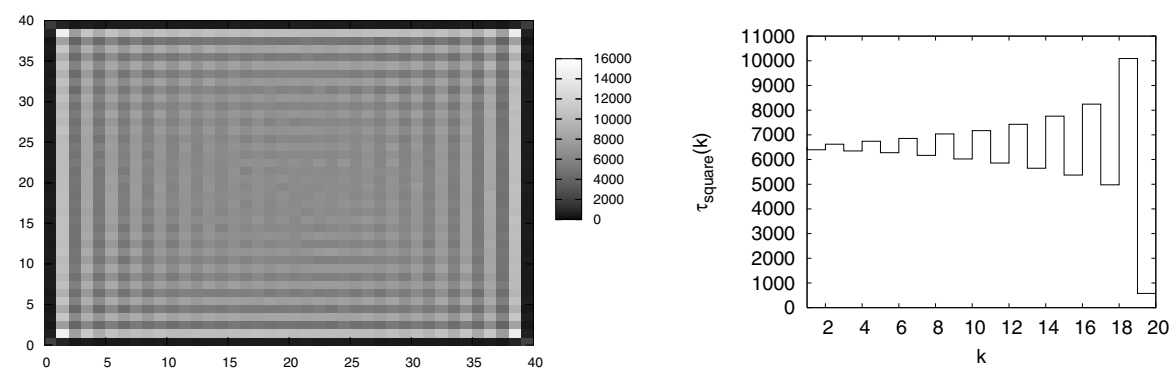

Fig. 2. Brood tending values $\tau$; dark (resp. light) colors correspond to small (resp. large) values (left); mean brood tending $\tau_{\text {square }}(t, k), 1 \leq k \leq 20$ (right); parameters were $t=20000, p=0.05$, and $\beta=\infty$ 
we measured the brood care in every single cell (see in Figure 21). Suppose a $\beta$ value is used, that combines a random behavior with the deterministic behavior (comp. Equation [1). As in the model of [7] there is a ring of stationary ants on the boundary. The influence of the deterministic behavior of the ants will lead to the effect, that ants which occur on the boundary tend to move towards the center. Therefore, the brood care in the ring of cells that abut on the boundary will be bad. The brood care in the next ring of cells towards the center will be better, this effect continues until the random influence gets too large, and brood care is more evenly distributed. The brood care after 20000 steps is shown for $\beta=\infty$ in Figure 2, the brood care values $\bar{\tau}_{\text {square }}$ are also given. Obviously the uneven brood care will lead to smaller $\sigma^{*}$ values in the center of the nest.

\section{Results for the Extended Model}

In Figure 3 the tending behavior in the extended model is shown for $p=0.05$, $f=0.03, \lambda=0.5$ and $\sigma=0.5$. This result is particularly interesting, as the used parameter for the normal distribution of $\mathrm{CO}_{2}$ in the nest leads approximately to the equilibrium distribution as given in [1] and can therefore be considered as a realistic distribution. In Figure 3 three different regions of brood tending can be distinguished. This helps to explain why the brood is organized in concentric rings around the nest center. To examine the brood tending (resp. brood feeding) behavior more exactly the average intensity of tending (resp. feeding) in concentric circles and squares around the center was measured (see Figure 4). Again, the different regions can be observed.

Brood tending results for different driving rates $p \in\{0.01,0.03,0.1\}$ are shown in Figure 5. The existence of different brood tending areas is obviously quite robust with respect to the driving rate. The influence of the degree of randomness in the ants behavior is much stronger, as can be seen in Figure 6. While for $\lambda=0.9$ (nearly random movements of the ants) the smallest brood tending values are in the nest center, this is very different for a value of $\lambda=0.7$, where three different tending areas appear. For even smaller values of $\lambda$ the tending in the nest center becomes extremely large and does not model realistic ant tending behavior $(\lambda=0.2$ in our simulation).

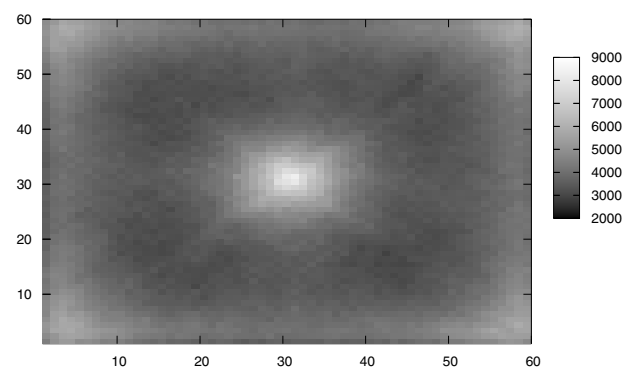

Fig. 3. Brood tending values $\tau$ after $t=20000$ steps; parameters were $p=0.05$, $\sigma=0.5, \lambda=0.5$, and $f=0.03$ 

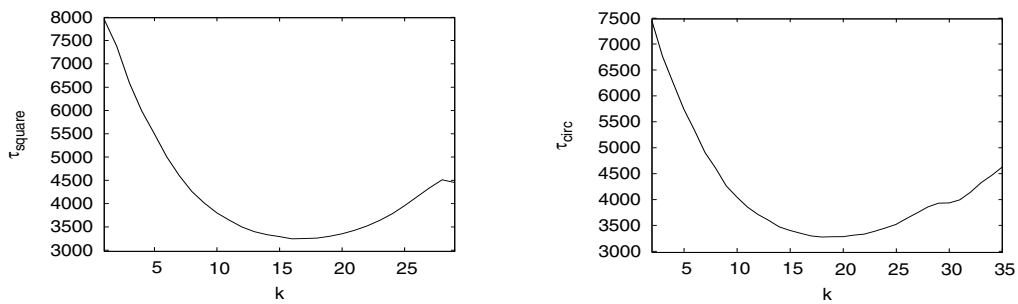

Fig. 4. Mean brood tending values $\bar{\tau}_{\text {square }}(t, k)$ (left) and $\bar{\tau}_{\text {circ }}(t, k)$ (right) for test run shown in Figure 3
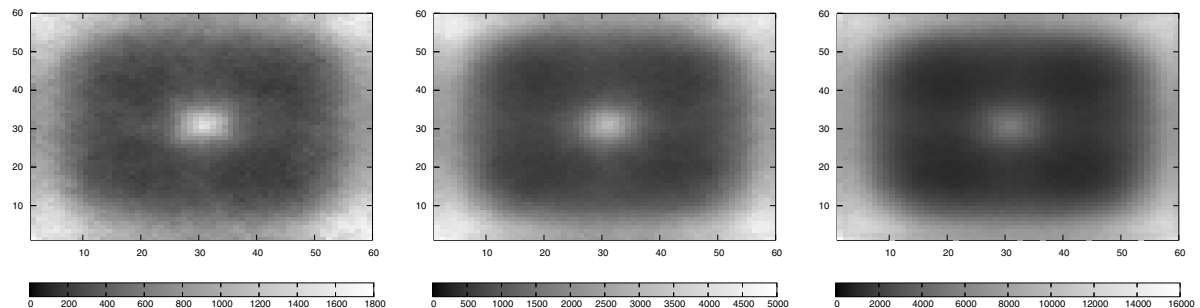

Fig. 5. Brood tending values $\tau$ for different driving rates $p=0.01$ (left) $p=0.03$ (middle), and $p=0.1$ (right) after $t=10000$ steps; parameters were $\sigma=0.5, \lambda=0.7$, and $f=0.02$
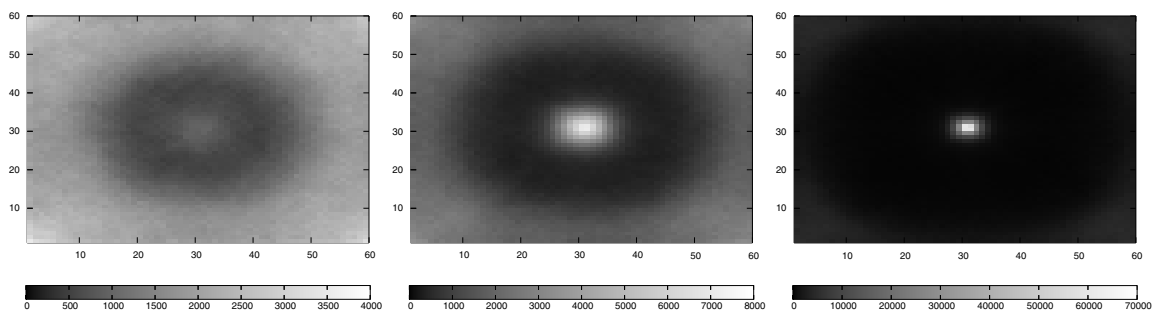

Fig. 6. Brood tending values $\tau$ for different degrees of randomness after 10000 steps; parameters were $\lambda=0.9$ (left), $\lambda=0.7$ (middle), $\lambda=0.2$ (right), $p=0.05, f=0.008$, $\sigma=0.25$

Due to space limitation we can only present the following results. Hence, results for other nest sizes or for the second strategy to combine strict gradient movement with randomness have to be omitted. Similar to [1] we investigated the brood tending behavior for situations with a different distribution of $\mathrm{CO}_{2}$. Such distributions can occur when, e.g., only three sides of the nest are open, and the other side is not a $\mathrm{CO}_{2}$ sink. This leads to a different equilibrium distribution of $\mathrm{CO}_{2}$ and influences the behavior of the ants. Figure 7 shows the brood feeding and tending behavior for this case (up, left, and bottom) and a $\mathrm{CO}_{2}$ distribution, where the largest concentration is not located in the center of the nest, but in 

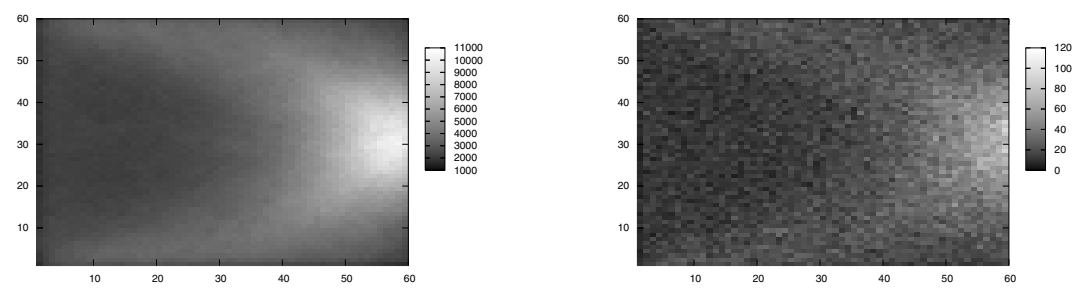

Fig. 7. Brood tending $\tau$ (left) and feeding $\zeta$ (right) values for three open nest sides (up, left, and bottom) and a $\mathrm{CO}_{2}$ distribution, where the largest concentration is located in the middle of the right border; parameters were $p=0.05, \lambda=0.5, f=0.02, \sigma=1$, and $t=10000$

the middle of the right border. It would be interesting to compare this results with experiments for such a situation with real ants.

\section{Conclusion}

In this paper a cellular automata model for ant brood tending behavior that uses a combination of deterministic and stochastic behavior was reexamined. We have shown that uneven brood care is an artifact of the model. A modification of this model has been introduced where uneven brood tending patterns occurs similar to those observed in real ant colonies. The model has also been extended to include the carbon dioxide distribution that influences the ants movement behavior and to model food delivering by the ants. Our results may help to explain the existence of pattern formation in real ant nests.

\section{References}

1. M.D. Cox and G.B. Blanchard. Gaseous templates in ant nests. Journal of Theoretic Biology, 204:223-238, 2000.

2. L. Deneubourg, S. Goss, N. Franks, A.B. Sendova-Franks, C. Detrain, and L. Chretien. The dynamics of collective sorting: Robot-like ants and ant-like robots. In Proc. of the 1st Int. Conf on Simulation of Adaptive Behavior, pages 356-363, 1991.

3. N.R. Franks and A.B. Sendova-Franks. Brood sorting by ants: distributing the workload over the work-surface. Behavioral Ecology and Sociobiology, 30:109-123, 1992.

4. D. Grünbaum. Translating stochastic density-dependent individual behavior to a continuum model of animal swarming. J. of Mathematical Biology, 33:139-161, 1994.

5. H.J. Jensen. Lattice gas model of $1 / f$ noise. Phys. Rev. Lett., 64:3103-3106, 1990.

6. G. Nicolas and D. Sillans. Immediate and latent effects of carbon dioxide on insects. Annual Review of Entomology, 34:97-116, 1989.

7. D.V. O'Toole, P.A. Robinson, and M.R. Myerscough. Self-organized criticality in ant brood tending. Journal of Theoretical Biology, 221:1-14, 2003.

8. A.B. Sendova-Franks and J.V. Lent. Random walk models of worker sorting in ant colonies. Journal of Theoretical Biology, 217:255-274, 2002. 